\title{
Chemosensitivity test for 5-fluorouracil and 5-chloro-2, 4-dihydroxypyridine predicts outcome of gastric cancer patients receiving S-1 postoperatively
}

\author{
Kentaro Maejima $^{1}$, Akira Tokunaga ${ }^{1}$, Teruo Kiyama ${ }^{2}$, Hitoshi Kanno ${ }^{2}$, Hideki Bou ${ }^{1}$, Masanori Watanabe ${ }^{1}$, \\ HideYUKi SUZUKI ${ }^{1}$, and EIJI UCHIDA ${ }^{2}$ \\ ${ }^{1}$ Institute of Gastroenterology, Nippon Medical School Musashi Kosugi Hospital, 1-396 Kosugi-cho, Nakahara-ku, Kawasaki, Kanagawa \\ 211-8533, Japan \\ ${ }^{2}$ Department of Surgery, Nippon Medical School, Tokyo, Japan
}

\begin{abstract}
Background. Chemosensitivity tests have long been discussed but remain a topic of research. In this study, we investigated the correlation between the results of a chemosensitivity test for 5-fluorouracil and 5-chloro-2, 4-dihydroxypyridine and the clinical outcomes of gastric cancer patients treated with $\mathbf{S - 1}$, an oral fluoropyrimidine, as adjuvant chemotherapy.

Methods. For gastric cancer patients, we performed surgical treatment and a lymph node dissection of D2 or more. Afterwards, a chemosensitivity test for 5-fluorouracil and 5-chloro2, 4-dihydroxypyridine was performed, using the collagen gel droplet embedded culture drug-sensitivity test (CD-DST), in surgical specimens. All the patients received postoperative adjuvant chemotherapy with S-1 for 1 year, and the overall survival (OS), relapse-free survival (RFS), and adverse events were investigated.

Results. The chemosensitivity test was performed for 27 patients. The growth inhibition rate (IR) was $50 \%$ or more (high-sensitivity group) in $\mathbf{5 9 . 3} \%$ (16 cases) and it was under $50 \%$ (low-sensitivity group) in $40.7 \%$ (11 cases). The 3 -year OS rate was $100 \%$ in the high-sensitivity group and $62.34 \%$ in the low-sensitivity group. The 3-year RFS rate was $83.33 \%$ in the high-sensitivity group and $\mathbf{2 4 . 2 4} \%$ in the low-sensitivity group. Thus, the 3-year OS rate and the 3-year RFS rate were higher in the high-sensitivity group than in the low-sensitivity group. No adverse events of grade 3 or greater severity were observed.

Conclusions. The results of the chemosensitivity test were correlated with the patient outcome. Therefore, such results might be useful for individualizing cancer chemotherapy and for determining future indications for postoperative adjuvant chemotherapy.
\end{abstract}

Key words Chemosensitivity testing $\cdot \mathrm{CD}$-DST $\cdot$ Gastric cancer - Adjuvant chemotherapy $\cdot$ S-1

Offprint requests to: $\mathrm{K}$. Maejima

Received: February 4, 2010 / Accepted: June 17, 2010

\section{Introduction}

The selection of appropriate anticancer drugs for individual patients is important, and the recent development of numerous new anticancer drugs for the treatment of gastric cancer has increased expectations for improved therapeutic outcomes. Consequently, chemosensitivity tests have been accepted as a highly advanced medical technology, and their usefulness has been examined in nationwide clinical studies [1].

Individuals with tumors of a similar histopathological class can have markedly different clinical drug sensitivity profiles. In vitro drug sensitivity tests have thus been developed to individualize chemotherapy for cancer patients $[2,3]$.

Of note, a phase III study of surgical treatment alone vs adjuvant S-1 chemotherapy in patients with curative resections of stage II and stage III gastric cancers (Adjuvant Chemotherapy Trial of TS- 1 for Gastric Cancer [ACTS-GC]) reported that postoperative adjuvant chemotherapy with S-1 prolonged the survival of stage II and stage III gastric cancer patients [4]. In the present study, we investigated the predictive clinical value of a chemosensitivity test for 5-fluorouracil (5-FU) and 5-chloro-2, 4-dihydroxypyridine (CDHP) in patients receiving S-1 postoperatively. The study was performed in May 2004 at an institution affiliated with the Department of Surgery, Nippon Medical School.

Patients, materials, and methods

\section{Patients}

This study included 65 patients with stage II, III, or IV gastric cancer with curability A or B who had undergone surgical treatment and a lymph node dissection of D2 or more. The 65 patients were divided into a sensitivity-testing group and a non-test group, using the 
randomized clinical trial-envelope protocol. All the patients started adjuvant chemotherapy within 6 postoperative weeks. All the patients received two oral doses of $40 \mathrm{mg}$ of S-1 per square meter of body-surface area per day for 4 weeks, followed by 2 weeks of no chemotherapy. Specifically, during the treatment weeks, patients with a body-surface area of less than $1.25 \mathrm{~m}^{2}$ received $80 \mathrm{mg}$ daily; those with a body-surface area of $1.25 \mathrm{~m}^{2}$ or more but less than $1.5 \mathrm{~m}^{2}$ received $100 \mathrm{mg}$ daily; and those with a body-surface area of $1.5 \mathrm{~m}^{2}$ or more received $120 \mathrm{mg}$ daily. Patients underwent hematologic testing and assessments of clinical symptoms every 2 weeks. The presence of a relapse was determined using imaging studies, including ultrasonography, computed tomography (CT), gastrointestinal radiography series, and endoscopy. Patients underwent at least one type of imaging study, usually CT, at 6-month intervals during the first 2 years after surgery and at 1-year intervals thereafter until 5 years after surgery. The treatment was administered for 1 year postoperatively. The primary endpoint was the overall survival (OS) period, and the secondary endpoints were the relapse-free survival (RFS) period and the adverse events. Overall survival was defined as the period between operation and death. Relapse-free survival was defined as the period between operation and the first relapse.

\section{Drug sensitivity test}

In the sensitivity-testing group, the chemosensitivity test for 5-FU and CDHP was conducted using the collagen gel droplet embedded culture drug sensitivity test (CDDST) in surgically resected gastric cancer specimens. A portion of each tumor specimen was excised and thin slices were prepared. Each sample was treated with Dispersion Enzyme Cocktail EZ (Kurabo Industries, Osaka, Japan). The resulting cell suspension was inoculated into collagen-coated flasks (CG-flask; Kurabo Industries) and cultured in preculture medium (PCM-1; containing $10 \%$ fetal bovine serum [FBS]) at $37^{\circ} \mathrm{C}$ in $5 \% \mathrm{CO}_{2}$ overnight. Next, the collagen gel was digested with $0.05 \%$ collagenase (type I; Sigma-Aldrich Japan, Tokyo, Japan) and viable cancer cells were obtained. Type I collagen (Cellmatrix Type CD; Kurabo Industries), $10 \times \mathrm{F}-12$ medium, and a reconstitution buffer were mixed together in ice water at a ratio of $8: 1: 1$. The prepared cancer cell suspension was added to the collagen solution, with a final density of $1 \times 10^{5}$ cells $/ \mathrm{ml}$. Three drops of the collagen-cell mixture $(30 \mu \mathrm{l} /$ droplet $)$ were placed in each well of a six-well multiple plate on ice and allowed to gel at $37^{\circ} \mathrm{C}$ in a $\mathrm{CO}_{2}$ incubator; the final concentration was about $3 \times 10^{3}$ cells per collagen gel droplet. Dulbecco's Modified Eagle and F-12 medium (Gibco) containing $10 \%$ FBS was overlaid on each well $1 \mathrm{~h}$ later and incubated in a $\mathrm{CO}_{2}$ incubator at $37^{\circ} \mathrm{C}$ overnight.
Subsequently, the tumor cells in the collagen gel droplet were exposed to the treatment drugs at concentrations corresponding to the area under the drug concentration time curve (AUC) in clinical cases and incubated for $120 \mathrm{~h}$. The drug concentrations were $4.0 \mu \mathrm{g} / \mathrm{ml}$ of $5-\mathrm{FU}$ and $8.0 \mu \mathrm{g} / \mathrm{ml}$ of CDHP.After the removal of the medium containing the anticancer drugs, each well was rinsed twice with $3 \mathrm{ml}$ of Hanks' balanced salt solution (HBSS) each time, overlaid with $4 \mathrm{ml}$ of PCM-2 medium (serumfree medium; Kurabo Industries), and incubated for a further 7 days. At the end of the incubation, neutral red was added to each well at a final concentration of $50 \mu \mathrm{g} /$ $\mathrm{ml}$ and the colonies in the collagen gel droplets were stained for $2 \mathrm{~h}$. Each collagen droplet was fixed with $10 \%$ neutral-buffered formalin, washed in water, airdried, and quantified using image analysis. The growth rates of control incubations were calculated as the image density on day 7/image density on day 1 [5-8]. Cases with an inhibition rate of $50 \%$ or higher were judged as exhibiting high drug sensitivity.

\section{Statistical analysis}

All statistical analyses were performed using the JMP 8.0 statistical software program (SAS, Cary, NC, USA). The $\chi^{2}$ test was used for comparisons among groups. The 3 -year survival rates were estimated using the KaplanMeier method, and the log-rank test was used to compare the curves. $P$ values of less than 0.05 were considered statistically significant.

\section{Informed consent and approval}

All the patients were informed of their therapeutic options. None of the treatments were performed without first obtaining informed consent. The study protocol conformed to the ethical guidelines established by the Declaration of Helsinki and was approved by the institutional review board of Nippon Medical School.

\section{Results}

\section{Chemosensitivity test}

Of the 65 patients, 27 and 38 were included in the sensitivity-testing group and the no-test group, respectively. Based on the results of the sensitivity test, 16 and 11 of the 27 patients in the former group were judged as having high and low drug sensitivities, respectively. The growth inhibition rate was $50 \%$ or more (highsensitivity group) in $59.3 \%$ of the patients overall, and the mean overall growth inhibition rate was $56.39 \%$. No differences in age, histological type, stage, or curability were observed among the high-sensitivity group, the 
Table 1. Background factors of patients

\begin{tabular}{|c|c|c|c|c|}
\hline Factors & $\begin{array}{l}\text { High sensitivity } \\
\quad n=16\end{array}$ & $\begin{array}{l}\text { Low sensitivity } \\
\qquad n=11\end{array}$ & $\begin{array}{c}\text { Non-test } \\
n=38\end{array}$ & $P$ value \\
\hline Age (years) & $68.7 \pm 11.4$ & $70.4 \pm 9.0$ & $66.6 \pm 9.9$ & 0.4115 \\
\hline Sex (male:female) & $15: 1$ & $8: 3$ & $24: 14$ & 0.0425 \\
\hline Histology (tub:por:sig:muc:pap) & $9: 6: 0: 0: 1$ & $6: 5: 0: 0: 0$ & $15: 18: 2: 3: 0$ & 0.3173 \\
\hline Stage (II:IIIA:IIIB:IV) & $3: 5: 6: 2$ & $1: 3: 4: 3$ & $18: 8: 8: 4$ & 0.1561 \\
\hline Curability $(\mathrm{A}: \mathrm{B})$ & $4: 12$ & $2: 9$ & $15: 23$ & 0.3037 \\
\hline Average inhibition rate & $68.6 \pm 10.7 \%$ & $38.6 \pm 13.7 \%$ & - & $<0.01$ \\
\hline
\end{tabular}

tub, tubular adenocarcinoma; por, poorly differentiated adenocarcinoma; sig, signet-ring cell carcinoma; muc, mucinous adenocarcinoma; pap, papillary adenocarcinoma

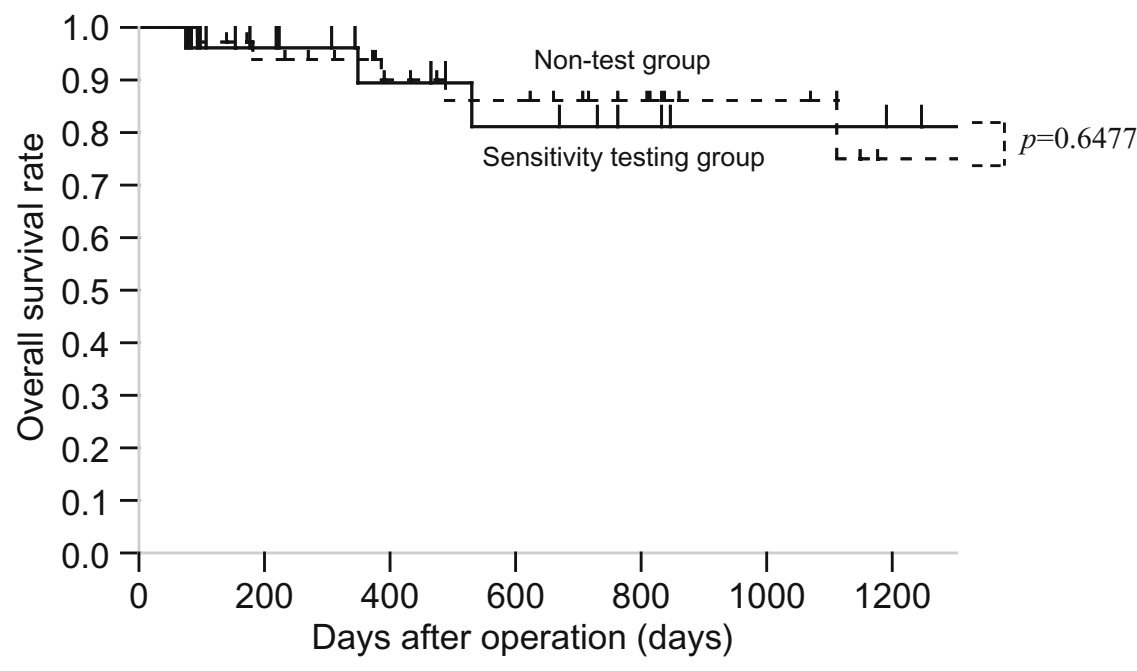

Fig. 1. The 3-year overall survival (OS) rate was $81.17 \%$ in the sensitivity-testing group and $85.88 \%$ in the non-test group low-sensitivity group, and the no-test group. In the high-sensitivity group, many of the patients were male (Table 1). The mean growth inhibition rates were $68.6 \pm$ $10.7 \%$ in the high-sensitivity group and $38.6 \pm 13.7 \%$ in the low-sensitivity group $(P<0.01)$.

\section{Clinical outcomes of the patients}

The median time from operation to follow-up was 844 days. The 3 -year OS rate was $81.17 \%$ in the sensitivitytesting group and $85.88 \%$ in the non-test group $(P=$ 0.6477 ; Fig. 1). The 3 -year RFS rate was $45.39 \%$ in the sensitivity-testing group and $64.31 \%$ in the non-test group ( $P=0.2685$; Fig. 2$)$. No differences in the 3 -year OS rate or the 3-year RFS rate were observed between the sensitivity-testing group and the non-test group. Within the sensitivity-testing group, the 3-year OS rate was $100 \%$ in the high-sensitivity group and $62.34 \%$ in the low-sensitivity group ( $P=0.0464$; Fig. 3$)$. The 3-year RFS rate was $83.33 \%$ in the high-sensitivity group and $24.24 \%$ in the low-sensitivity group ( $P=0.0117$; Fig. 4$)$. Thus, the 3-year OS rate and the 3-year RFS rate were significantly higher in the high-sensitivity group than in the low-sensitivity group. The mean IR in the patients who relapsed and died was relatively low (29.3\%).

\section{Toxicities}

All 65 patients were analyzed for adverse events. The grades of adverse events were defined according to the Common Terminology Criteria for Adverse Events, version 3.0 (CTCAE). No adverse events of grade 3 or greater severity were observed (Table 2).

\section{Discussion}

In the present study, the results of the chemosensitivity test for 5-FU and CDHP were clearly correlated with the outcome of patients receiving S-1 postoperatively. In this study, patients were divided into a high-sensitivity group and a low-sensitivity group based on the IR of the chemosensitivity test. Significant differences in the OS and RFS were obtained between the high-sensitivity and low-sensitivity groups when IR cutoff values of $20 \%$ and $50 \%$ were used, respectively (Table 3 ). However, because the number of patients and the background factors of the patients in each group differed when a cutoff value of $20 \%$ was used, we chose $50 \%$ as the cutoff value.

Two clinical studies in Japan, the Japanese Clinical Oncology Group (JCOG) 8801 study (mitomycin C 

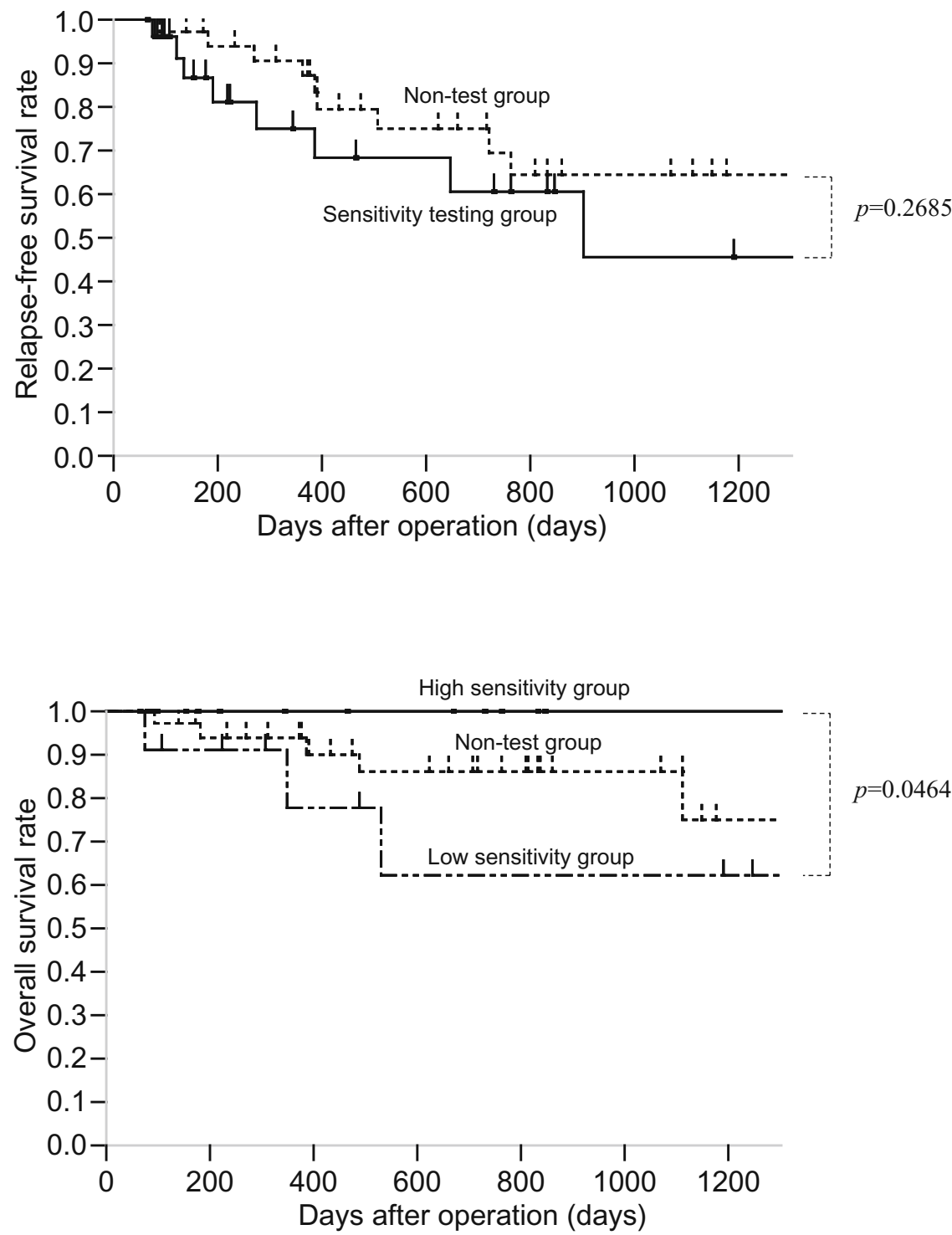

Fig. 2. The 3-year relapse-free survival (RFS) rate was $45.39 \%$ in the sensitivitytesting group and $64.31 \%$ in the non-test group
Fig. 3. The 3 -year OS rate was $100 \%$ in the high-sensitivity group and $62.34 \%$ in the low-sensitivity group

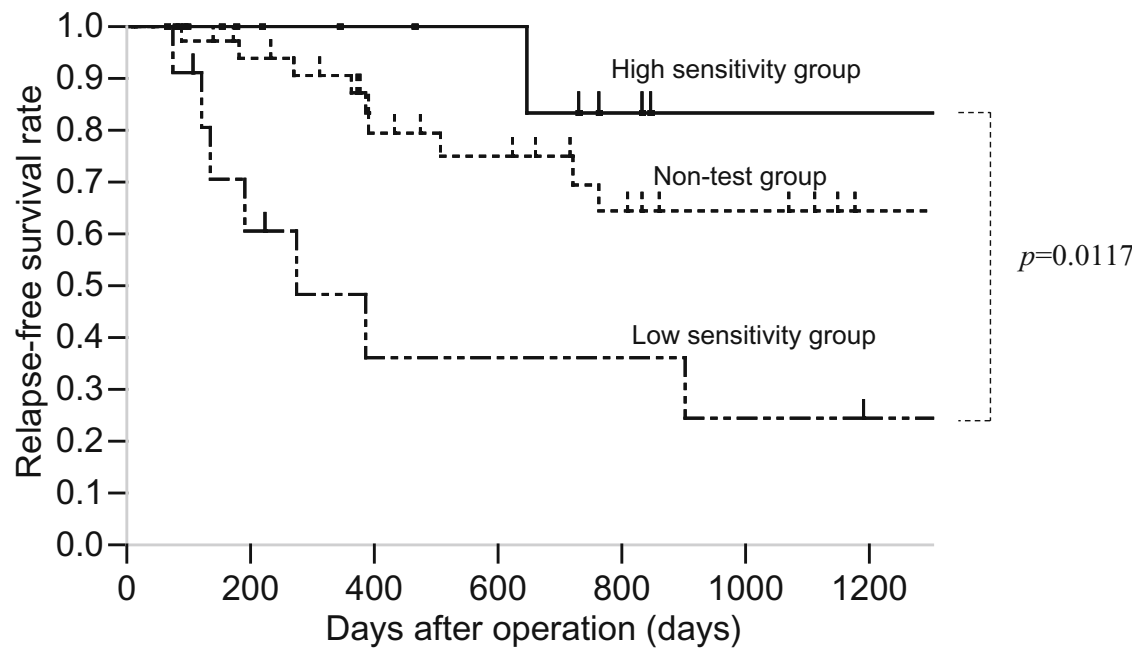

Fig. 4. The 3-year RFS rate was $83.33 \%$ in the high-sensitivity group and $24.24 \%$ in the low-sensitivity group 
Table 2. Adverse events according to treatment

\begin{tabular}{lcc}
\hline Event & $\begin{array}{c}\text { Adjuvant } n=65 \\
(\%)\end{array}$ & $\begin{array}{c}\text { Above grade 3 } \\
(\%)\end{array}$ \\
\hline Leukopenia & 6.2 & 0 \\
Neutropenia & 3.1 & 0 \\
Anemia & 3.1 & 0 \\
Thrombocytopenia & 6.2 & 0 \\
Elevated AST·ALT level & 1.6 & 0 \\
Elevated total serum bilirubin level & 0 & 0 \\
Stomatitis & 3.1 & 0 \\
Pigmentation & 1.6 & 0 \\
Exanthema & 0 & 0 \\
Anorexia & 4.6 & 0 \\
Nausea·vomitus & 1.6 & 0 \\
Diarrhea & 0 & 0 \\
\hline Grades of & 0 & 0
\end{tabular}

Grades of adverse events were defined according to the Common Terminology Criteria for Adverse Events (CTCAE) version 3.0

AST, aspartate aminotransferase; ALT, alanine aminotransferase

Table 3. Ratios of high- and low-sensitivity groups, and $P$ values of OS and RFS by log-rank test determined at various IR cutoff values

\begin{tabular}{lcccc}
\hline $\begin{array}{l}\text { IR cutoff } \\
(\%)\end{array}$ & $\begin{array}{c}\text { High sensitivity } \\
(n)\end{array}$ & $\begin{array}{c}\text { Low sensitivity } \\
(n)\end{array}$ & OS $P$ value & RFS $P$ value \\
\hline 20 & 25 & 2 & 0.0002 & 0.0100 \\
30 & 24 & 3 & 0.1119 & 0.3816 \\
40 & 23 & 4 & 0.0097 & 0.1423 \\
50 & 16 & 11 & 0.0464 & 0.0117 \\
60 & 12 & 15 & 0.1583 & 0.1504 \\
70 & 8 & 19 & 0.2190 & 0.3423 \\
80 & 2 & 25 & 0.3280 & 0.2252 \\
\hline
\end{tabular}

IR, inhibition rate; OS, overall survival; RFS, relapse-free survival

$[\mathrm{MMC}]+5-\mathrm{FU}+$ uracil and tegafur [UFT] vs surgical treatment alone) [9] and the JCOG9206-1 study (mitomycin, fluorouracil, and cytosine [MFC] + 5-FU vs surgical treatment alone) [10], failed to confirm the usefulness of postoperative adjuvant chemotherapy. However, in a post-stratification analysis of the JCOG8801 study, the 5-year survival rate was found to be $78.2 \%$ for pT2,3 and $\mathrm{pN}(+)$ patients in the adjuvant chemotherapy group, while the rate was $66.6 \%$ in the pT2,3 and pN (+) patients treated with surgery alone. Thus, adjuvant chemotherapy appeared to exert some efficacy [11]. In the National Surgical Adjuvant Study Group for Gastric Cancer (NSAS-GC) trial, which was initiated based on data from the post-stratification analysis, surgical treatment alone was compared with the oral administration of UFT in pT2 and pN1,2 patients, and prolonged survival was confirmed in the UFT group [12]. A phase III study comparing the oral administration of S-1 and surgical treatment alone (ACTS-GC) was also conducted in stage II and stage III gastric cancer patients who had undergone curative resections, and a prolongation of survival was confirmed in the S-1 group. At present, oral $\mathrm{S}-1$ is the standard postoperative adjuvant chemotherapy for gastric cancer in Japan [4]. Of note, although many in vitro and in vivo methods have been reported to predict the clinical chemosensitivity of tumors in humans $[13,14]$, their clinical usefulness has not yet been established. However, in joint studies conducted between 1990 and 1993, MMC + UFT was used as the postoperative adjuvant chemotherapy regimen in patients with advanced gastric cancer. Based on the results of chemosensitivity tests conducted in surgically resected specimens, the OS was compared between the group in which the tumor was found to be sensitive to MMC or 5-FU (sensitivity group) and the group in which the tumor was resistant to either drug (nonsensitivity group); the OS rate was found to be significantly higher in the sensitivity group than in the non-sensitivity group [15]. Other reports have also shown significantly more favorable survival periods following adjuvant chemotherapy among patients judged to exhibit sensitivity to the anticancer drugs, compared with patients who did not exhibit sensitivity, according to inferential statistics [16-23]. In addition, similar results have been reported for patients with other types of cancer $[24,25]$. Nevertheless, the accuracy of assays for predicting survival needs to be validated in a prospective clinical trial involving patients who have 
received the drugs in a postoperative adjuvant setting [26]. Consequently, the validity of sensitivity testing was investigated in the present study by conducting chemosensitivity testing in patients who had received adjuvant chemotherapy with S-1 (i.e., the standard postoperative adjuvant chemotherapy for gastric cancer at present in Japan). The present results revealed that patients with high drug sensitivity had a prolonged survival period and prolonged relapse-free survival (RFS) period, compared with patients who had low drug sensitivity.

In a study reported in 1994 that included stage III and stage IV gastric cancer patients treated with postoperative adjuvant chemotherapy and in whom chemosensitivity testing had been performed, the survival rate in the sensitivity group was significantly more favorable than that in the non-sensitivity group; conversely, no significant difference in the survival rate was observed between the non-sensitivity group and the surgical treatment-alone group [16]. In other words, the postoperative adjuvant chemotherapy might not have had an effect on survival among the patients who were judged as having poor drug sensitivity, and these patients were thus exposed unnecessarily to the risk of adverse reactions associated with chemotherapy. The chemotherapy treatment was also expensive and time-consuming. At present in Japan, S-1 therapy is used as postoperative adjuvant chemotherapy for all patients with stage II and stage III gastric cancer undergoing surgery, based on the results of the ACTS-GC study. However, the chemotherapy might possibly be ineffective and not required in some of these patients. Further investigation using a randomized controlled trial (RCT) is needed. Also, a previous report has suggested that making decisions regarding adjuvant chemotherapy based on the results of chemosensitivity testing could be economically effective in medical settings, allowing medical care costs associated with unnecessary chemotherapy to be avoided [27], thereby suggesting the future usefulness of chemosensitivity tests. On the other hand, several problems remain to be resolved. At present, the chemosensitivity test (CD-DST) for gastric cancer has been approved for health insurance reimbursement in Japan, but the indications for CD-DST are restricted to curability $\mathrm{C}$ gastric cancer. However, the CD-DST might also be useful for patients with curability A and B cancers who are scheduled to receive adjuvant chemotherapy. Furthermore, the existing chemosensitivity testing method requires a certain amount of tissue and can be difficult to perform using biopsy materials. A sensitivity-testing method that can be used with biopsy specimens is needed for patients with unresectable gastric cancer. Such tests might improve the therapeutic outcome and allow for individualized treatment by leading to the development of solutions to overcome these problems and tasks.
Acknowledgments The authors are greatly indebted to former Professor Tetsuro Kubota of Keio University for his generous and valuable advice in the present study.

\section{References}

1. Kubota T. Individualized tumor response assay (in Japanese). Jpn J Cancer Chemother 2008;35:174-7.

2. Hoffman RM. In vitro sensitivity assays in cancer: a review, analysis and prognosis. J Clin Lab Anal 1991;5:133-43.

3. Hoffman RM. In vitro assays for chemotherapy sensitivity. Crit Rev Oncol Hematol 1993:15:99-111.

4. Sakuramoto S, Sasako M, Yamaguchi T, Kinoshita T, Fujii M, Nashimoto A, et al. Adjuvant chemotherapy for gastric cancer with S-1, an oral fluoropyrimidine. N Engl J Med 2007;357: 1810-20.

5. Kobayashi H. Development of a new in vitro chemosensitivity test using collagen gel droplet embedded culture and image analysis for clinical usefulness. Recent Results Cancer Res 2003;161: 48-61.

6. Kobayashi H, Higashiyama M, Minamigawa K, Tanisaka K, Takano $\mathrm{T}$, Yokouchi $\mathrm{H}$, et al. Examination of in vitro chemosensitivity test using collagen gel droplet culture method with colorimetric endpoint quantification. Jpn J Cancer Res 2001;92:203-10.

7. Hanatani Y, Kobayashi H, Kodaira S, Takami H, Asagoe T, Kaneshiro E. An in vitro chemosensitivity test for gastric cancer using collagen gel droplet embedded culture. Oncol Rep 2000; 7:1027-33.

8. Mori S, Kunieda K, Sugiyama Y, Saji S. Prediction of 5-fluorouracil and cisplatin synergism for advanced gastrointestinal cancers using a collagen gel droplet embedded culture. Surg Today 2003;33:577-83.

9. Nakajima T, Nashimoto A, Kitamura M, Kito T, Iwanaga T, Okabayashi K, et al. Adjuvant mitomycin and fluorouracil followed by oral uracil plus tegafur in serosa-negative gastric cancer: a randomized trial. Lancet 1999;354:273-7.

10. Nashimoto A, Nakajima T, Furukawa H, Kitamura M, Kinoshita T, Yamamura Y, et al. Randomized trial of adjuvant chemotherapy with mitomycin, fluorouracil, and cytosine arabinoside followed by oral fluorouracil in serosa-negative gastric cancer: Japan Clinical Oncology Group 9206-1. J Clin Oncol 2003;21:2282-7.

11. Kitamura M, Nakajima T, Ohta K, Nashimoto A, Yamamura Y, Furukawa $\mathrm{H}$, et al. Adjuvant chemotherapy of gastric cancer: JCOG experience. New York Heidelberg Berlin Tokyo: Springer; 1999. p. 32-41.

12. Kinoshita T, Nakajima T, Ohashi Y. Adjuvant chemotherapy with uracil-tegafur for serosa-negative advanced gastric cancer. Results of a randomized trial by National Surgical Adjuvant Study of Gastric Cancer. 2005 ASCO Annual Meeting Proceedings. J Clin Oncol 2005;16S:4021.

13. Hamburger AW, Salmon SE. Primary bioassay of human tumor stem cells. Science 1977;197:461-3.

14. Von Hoff DD. He's not going to talk about in vitro predictive assays again, is he? J Natl Cancer Inst 1990;82:96-101.

15. Kubota T, Sasano N, Abe O, Nakao I, Kawamura E, Saito T, et al. Potential of the histoculture drug-response assay to contribute to cancer patient survival. Clin Cancer Res 1995;1:1537-43.

16. Saikawa Y, Kubota T, Furukawa T, Suto A, Watanabe M, Kumai $\mathrm{K}$, et al. Single-cell suspension assay with an MTT end point is useful for evaluating the optimal adjuvant chemotherapy for advanced gastric cancer. Jpn J Cancer Res 1994;85:762-5.

17. Furukawa T, Kubota T, Hoffman RM. Clinical applications of the histoculture drug response assay. Clin Cancer Res 1995;1: 305-11.

18. Fujita K, Kubota T, Matsuzaki SW, Otani Y, Watanabe M, Teramoto T, et al. Further evidence for the value of the chemo- 
sensitivity test in deciding appropriate chemotherapy for advanced gastric cancer. Anticancer Res 1998;18:1973-8.

19. Abe S, Kubota T, Matsuzaki SW, Otani Y, Watanabe M, Teramoto $\mathrm{T}$, et al. Chemosensitivity test is useful in evaluating the appropriate adjuvant cancer chemotherapy for stage III non-scirrhous and scirrhous gastric cancers. Anticancer Res 1999;19:4581-6.

20. Kubota T, Egawa T, Otani Y, Furukawa T, Saikawa Y, Yoshida M, et al. Cancer chemotherapy chemosensitivity testing is useful in evaluating the appropriate adjuvant cancer chemotherapy for stages III/IV gastric cancer without peritoneal dissemination. Anticancer Res 2003;23:583-7.

21. Nakamura R, Saikawa Y, Kubota T, Kumagai A, Kiyota T, Ohashi M, et al. Role of the MTT chemosensitivity test in the prognosis of gastric cancer patients after postoperative adjuvant chemotherapy. Anticancer Res 2006;26:1433-7.

22. Iwahashi M, Nakamori M, Nakamura M, Noguchi K, Ueda K, Nakatani Y, et al. Individualized adjuvant chemotherapy guided by chemosensitivity test sequential to extended surgery for advanced gastric cancer. Anticancer Res 2005;25:3453-60.
23. Kubota T, Weisenthal L. Chemotherapy sensitivity and resistance testing: to be "standard" or to be individualized, that is the question. Gastric Cancer 2006;9:82-7.

24. Fujita Y, Hiramatsu M, Kawai M, Nishimura H, Miyamoto A, Tanigawa N. Histoculture drug response assay predicts the postoperative prognosis of patients with esophageal cancer. Oncol Rep 2009;21:499-505.

25. Yamaue $H$, Tani $M$, Onishi $H$, Kinoshita $H$, Nakamori $M$, Yokoyama S, et al. Locoregional chemotherapy for patients with pancreatic cancer: intra-arterial adjuvant chemotherapy after pancreatectomy with portal vein resection. Pancreas 2002;25: 366-72.

26. Kodera Y, Ito S, Fujiwara M, Mochizuki Y, Ohashi N, Ito Y, et al. In vitro chemosensitivity test to predict chemosensitivity for paclitaxel, using human gastric carcinoma tissues. Int J Clin Oncol 2006;11:449-53.

27. Kubota T. Individualized tumor response assay. Nippon Rinsho 2008;66:239-44. 